\title{
ON AN INEQUALITY FOR THE ENTROPY OF A PROBABILITY DISTRIBUTION
}

\author{
Cvetan Jardas, Josip PeČarić, Rajko RoKi AND Nikola SARAPA
}

Abstract. In this paper we prove that Alzer's inequality for the entropy of a probability distribution (see [3]) is valid with reverse sign of the inequality. function.

Mathematics subject classification (2000): 26D15, 94A17, 28D20.

Key words and phrases: Entropy of probability distribution, inequality for infinite series, convex

\section{REFERENCES}

[1] J.-P. Allouche AND M. Mendes France, On an extremal property of the Rudin-Shapiro sequence, Mathematica 42 (1985), 33-38.

[2] J.-P. Allouche, M. Mendes France and G. Tenenbaum, Entropy: An inequality, Tokyo J. Math. 11, 2 (1988), 323-328.

[3] H. AlZER, Note on an inequality for infinite series, Acta Math. Hungar. 67(3) (1995), $203-206$.

[4] Z. DARócZY, Inequalities for some infinite series, Acta Math. Hungar. 75(1-2) (1997), 27-30.

[5] Ž. PAUŠE, Uvod u teoriju informacije, 2. izd., Školska knjiga, Zagreb, 1989.

[6] A. RÉNYI, Wahrscheinlichkeitsrechnung (mit einem Anhang über Informationstheorie), VEB Deutscher Verlag der Wisssenschaften, Berlin, 1966. 